\title{
Epidermolysis bullosa simplex with mottled pigmentation: the first Slovenian case
}

\author{
Aleksandra Bergant Suhodolčan ${ }^{1 凶}$, Vlasta Dragoš $^{1}$
}

\begin{abstract}
Epidermolysis bullosa simplex with mottled pigmentation is a rare subtype of epidermolysis bullosa simplex that is characterized by nonscarring blistering and reticulated hyperpigmentation. We report the first Slovenian case of a newborn with blisters, who later presented with hyperpigmented macules in the first year of life. A missense p.Pro25Leu mutation in the KRT5 gene was confirmed.
\end{abstract}

Received: 14 January 2014 | Returned for modification: 11 February 2014 | Accepted: 10 March 2014

\section{Introduction}

Epidermolysis bullosa simplex (EBS) is a group of genetic diseases in which blisters occur spontaneously or after minor trauma. The blister is localized in the basal layer of epidermal cells, although the recent classification of EBS also includes three disorders that result in a plane of separation within the suprabasal epidermis (suprabasal EBS). Because of the characteristic level of cleavage, EBS is sometimes termed epidermolytic EBS. Most of the cases are inherited in an autosomal dominant mode, but cases of recessive EBS also exist. Table 1 presents classification of major types of EBS. The prevalence of different forms of EBS has not been systematically studied and can therefore only be estimated. The prevalence of localized EBS is estimated at five to 20 per million, whereas the prevalence of the generalized form is about two per million (1). In 2003, an epidemiological study of EBS in Slovenia was performed, and a prevalence of 14 per million was recorded. According to clinical manifestation, the patients had localized EBS (previously called Weber-Cockayne) and generalized EBS or other (previously called Köbner) variants. Molecular defects were detected in Keratin 14 in five patients and in Keratin 5 in two patients (2). So far, no EBS with mottled pigmentation in Slovenia has been described.

EBS with mottled pigmentation is a rare subtype of EBS and is usually autosomal dominant inherited, but a few "de novo" mutations have been described. It is characterized by nonscarring blistering and reticulated hyperpigmentation. We present a case of a "de novo" mutation in the KRT5 gene.

\section{Case report}

A 16-day-old boy presented to our clinic with blisters and crusts on his fingers and toes and erosions on the gluteus and around the navel. Blistering and crusts on the fingers were already observed in the first days following birth (Fig. 1). He had previously been treated for staphylococcal infection with a systemic antibiotic, without success. New blisters were forming and were healing without sequelae. Thorough physical examination did not reveal any other abnormalities. The family history was negative for skin diseases, including blistering. A biopsy of the lesion was performed at the age of 6 weeks. Histopathologic and electromicroscopic examination revealed vacuolae in the basal layer of keratinocytes and suggested a diagnosis of EBS. At the age of 6 months the patient presented with asymptomatic hyperpigmented macules on the extremities and later on the entire body, excluding the face (Fig. 2). This suggested a rare type of EBS with mottled pigmentation. Gene sequencing was performed when the patient was 11 months old. No DNA variants were noted in the Keratin 14 gene, but a pathogenic heterozygote Keratin 5 mutation KRT5:c.74C $>$ T variant (missense KRT5:p.Pro25Leu mutation) was identified, which means that the child is heterozygous for a $\mathrm{C}>\mathrm{T}$ nucleotide substitution in exon 1, resulting in the replacement of a proline codon with a leucine codon at amino acid position in the $\mathrm{KRT}_{5}$ gene. The lesions were treated with topical antibiotic. The parents were instructed to prevent potential trauma and infections.

Table 1 | Classification of major types of EBS (1).

\begin{tabular}{|c|c|c|c|}
\hline & Inheritance & Protein defect & Gene defect \\
\hline EBS localized (Weber-Cockayne) & $A D$ & Keratin 5, 14 & KRT5, KRT14 \\
\hline EBS generalized, other (Köbner) & $A D$ & Keratin 5, 14 & KRT5, KRT14 \\
\hline EBS Dowling-Meara & $A D$ & Keratin 5, 14 & KRT5, KRT14 \\
\hline EBS with mottled pigmentation & $A D$ & Keratin 5, 14 & KRT5, KRT14 \\
\hline Autosomal recessive EBS (not associated with muscular dystrophy) & $A R$ & Keratin 14 & KRT14 \\
\hline EBS with muscular dystrophy & AR & Plectin & PLEC1 \\
\hline EBS Ogna & $A D$ & Plectin & PLEC1 \\
\hline EBS superficialis & $A D$ & Unknown & Unknown \\
\hline Skin fragility-ectodermal dysplasia syndrome & $A R$ & Plakophilin-1 & PKP1 \\
\hline Lethal acantolytic EB & $\mathrm{AR}$ & Desmoplakin & DSP \\
\hline
\end{tabular}




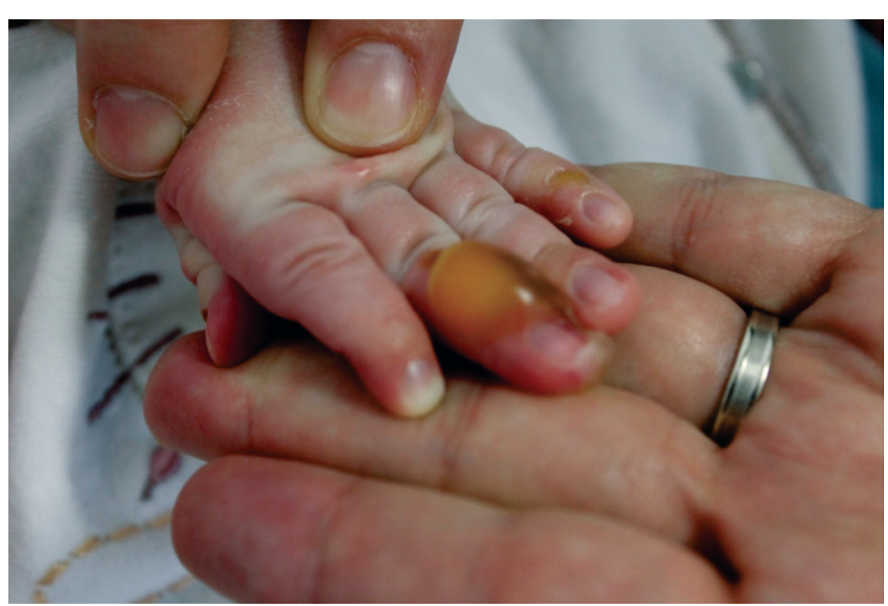

Figure 1 | Blisters on the hand (age: 3 weeks).

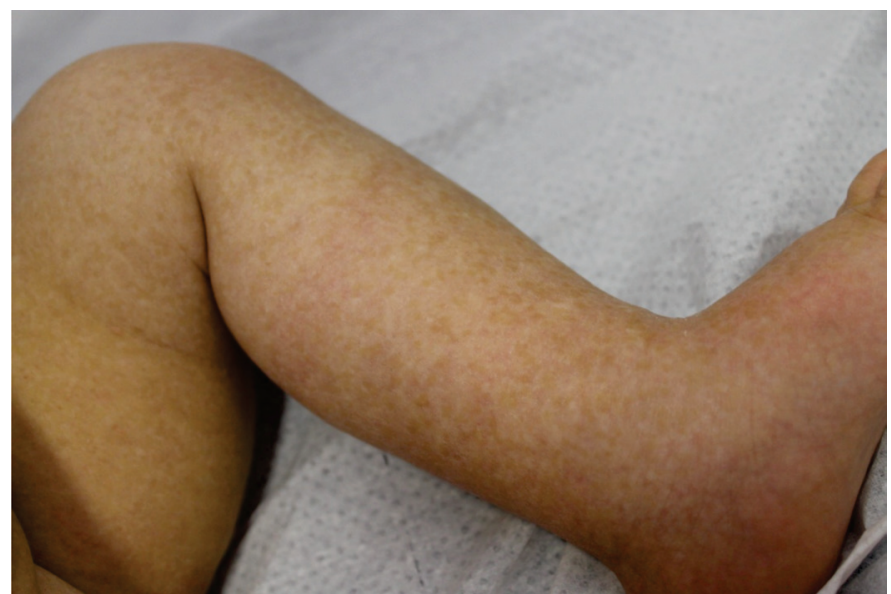

Figure 2 | Hyperpigmented macules on the lower extremities (age: 1 year).

\section{Discussion}

EBS is an inherited mechanobullous disorder characterized by skin fragility and blister formation following minor trauma of the

\section{References}

1. Irvine AD, Hoeger PH, Yan AC. Harper's textbook of pediatric dermatology. 3rd ed. West Sussex (UK): Wiley-Blackwell; c2011. Chapter 118, Epidermolysis bullosa; p.118.1-5.

2. Dragoš V, Podrumac B, Komel R, Liović M, Kansky A. Epidermolysis bullosa simplex in Slovenia. Acta Dermatovenerol Alp Panonica Adriat. 2003;12:83-8.

3. Fine JD. Inherited epidermolysis bullosa: recent basic and clinical advances. Curr Opin Pediatr. 2010;22:453-8.

4. Fischer T, Gedde-Dahl T Jr. Epidermolysis bullosa simplex and mottled pigmentation: a new dominant syndrome. I. Clinical and histological features. Clin Genet. 1979;15:228-38.

5. Echeverría-García B, Vicente A, Hernández A, Mascaró JM, Colmenero I, Terrón A, et al. Epidermolysis bullosa simplex with mottled pigmentation: a family report and review. Pediatr Dermatol. 2012; 30:e125-31.

6. Fuchs $\mathrm{E}$, Green $\mathrm{H}$. Changes in keratin gene expression during terminal differentiation of the keratinocyte. Cell. 1980;19:1033-42. skin (3). EBS with mottled pigmentation is a rare variant of EBS, first reported by Fischer and Gedd-Dahl in 1979, which presents with nonscarring blistering and slowly progressive reticulated hyperpigmentation (4). Multiple families and some sporadic cases have been reported all over the world. The blisters usually appear at birth and tend to decrease with age, only rarely appearing in adults. Hyperpigmentation usually begins later in infancy and childhood, making EBS with mottled pigmentation difficult to distinguish from other subtypes of EBS in the neonatal period. Adults can also develop punctate palmoplatar hyperkeratosis and nail dystrophy (5). The diagnosis is based on typical clinical findings, family history, gene mapping, and molecular analysis. Similar to other subtypes of EBS, mutations in EBS with mottled pigmentation are in the Keratin 5 and Keratin 14 genes and the intermediate filament (IF) proteins, expressed in basal keratinocytes in the epidermis and related complex epithelia (6). The mutation most commonly found in EBS with mottled pigmentation is the missense p.Pro25Leu mutation, which is also thought to be responsible for the aberrant pigmentation. There is growing evidence showing that keratin proteins functionally interact with melanin pigments, their malfunction resulting in aberrant melanosome uptake and consequently in hyperpigmented areas $(7,8)$.

\section{Conclusion}

The missense p.Pro25Leu mutation was confirmed in our patient. The case illustrates that a diagnosis of a phenotype of EBS cannot always be made as the first signs of the disease appear during the neonatal period, but prolonged follow-up and monitoring are required. Because there was no family history of blistering disease or hyperpigmentation now or in the past, we assume that the mutation appeared "de novo." Gene mapping of the parents could be performed to confirm this hypothesis. Sporadic "de novo" mutations on genes for Keratin 5 and 14 have been described previously $(9,10)$. However, this is the first confirmed Slovenian case of EBS with mottled pigmentation.

7. Irvine AD, Rugg EL, Lane EB, Hoare S, Peret C, Hughes AE, et al. Molecular confirmation of the unique phenotype of epidermolysis bullosa simplex with mottled pigmentation. Br J Dermatol. 2001;144:40-5.

8. Gu LH, Coulombe PA. Defining the properties of the nonhelical tail domain in type II keratin 5: insight from a bullous disease-causing mutation. Mol Biol Cell. 2005;16:1427-38.

9. Pascucci M, Posteraro P, Pedicelli C, Provini A, Auricchio L, Paradisi M, et al. Epidermolysis bullosa simplex with mottled pigmentation due to de novo $\mathrm{P} 25 \mathrm{~L}$ mutation in keratin 5 in an Italian patient. Eur J Dermatol. 2006;16:620-2.

10. Ołdak M, Przybylska D, Kosińska J, Federowicz A, Woźniak K, Płoski R, et al. Novel de novo mutation in KRT14 underlies a localized form of epidermolysis bullosa simplex. Eur J Dermatol. 2013;23:404-6. 